\title{
Oral propranolol therapy in 23 infants with infantile hemangioma
}

\author{
Jaeyoon Kim, Jong Won Hong, Tai Suk Roh, Won Jai Lee \\ Department of Plastic and Reconstructive Surgery, Institute for Human Tissue Restoration, Yonsei University College of Medicine, Seoul, Korea
}

Background Infantile hemangioma $(\mathrm{IH})$ is a common vascular tumor in pediatric patients, and is commonly treated with propranolol. We describe our experiences with dosage, response to treatment, and side effects in $23 \mathrm{IH}$ patients treated with propranolol.

Methods For this nonrandomized comparative cohort study, the authors enrolled 23 patients treated with propranolol. Photographs were taken before propranolol administration and at 3 , 6,9 , and 12 months after treatment. Treatment responses were objectively analyzed with a computer program.

Results There were three male and 20 female patients. Common tumor locations were the head and neck (13 cases, 56.5\%), trunk (four cases, 17.4\%), extremities (three cases, 13.0\%), and combined locations (three cases, 13.0\%). The response to propranolol was significantly lower in patients with two or more lesions than in patients with a single lesion in terms of both color fading $(P<0.001)$ and size reduction $(P<0.001)$. In male patients $(42.2 \pm 3.9)$, the change in a-values, indicating coloration, was higher than in female patients $(19.8 \pm 13.8)$ $(\mathrm{P}<0.001)$. In patients who started treatment before 6 months after birth, the size reduction was greater than in their counterparts (62.3\%; range, 3.0\%-93.0\% vs. $15.8 \%$; range, $1.0 \%$ $79.0 \% ; \mathrm{P}<0.001)$.

Conclusions Propranolol is an efficacious treatment with a good safety profile. In patients with a single lesion, the response to treatment was better in terms of color fading and size reduction. Furthermore, male patients responded better to propranolol treatment in terms of color fading than female patients, and starting treatment before 6 months after birth was more advantageous for size reduction.

Keywords Hemangioma / Propranolol / Therapeutics
Correspondence: Won Jai Lee Department of Plastic and Reconstructive Surgery, Institute for Human Tissue Restoration, Yonsei University College of Medicine, 50-1 Yonsei-ro, Seodaemun-gu, Seoul 03722, Korea

Tel: $+82-2-2228-2215$

Fax: +82-2-393-6947

E-mail: pswjlee@yuhs.ac

\section{INTRODUCTION}

Infantile hemangioma (IH) is a common vascular tumor in children. Schupp et al. [1] reported an estimated incidence rate in Asia of one in 125 persons annually. Female infants are more frequently affected than are male infants [2]. The disease is caused by an abnormal proliferation of endothelial cells of the capillary vessels and typically occurs at 3 to 4 weeks of age, peaking at 6 to 7 months of age, in the proliferative phase. After this period, the size of the tumor rarely decreases naturally, although its color can suddenly become less livid [3]. Most IHs are diagnosed via patient history and physical examination. In addition, imaging studies, such as Doppler ultrasound and magnetic resonance imaging (MRI), can characterize the location 
and extent of cutaneous and extracutaneous hemangiomas. MRI can also help differentiate IHs from high-flow vascular lesions such as arteriovenous malformations. Ultrasonography is useful for differentiating hemangiomas from other deep dermal or subcutaneous structures, such as cysts, pilomatrixomas, and lymph nodes [4].

Although hemangiomas are not routinely biopsied because the diagnosis can generally be made clinically, specimens may be evaluated for tissue-specific immunohistochemical markers, such as GLUT-1, merosin, Fc-gamma-RII, and Lewis Y antigens. These markers can differentiate IHs (positive staining for all markers) from other vascular neoplasms and malformations $[5,6]$.

Most IHs are small and self-limiting; however, complicated IHs with accompanying bleeding or infection may impact vital structures, such as the eye or airway, and medical or surgical treatment is therefore indicated in such cases [7]. Furthermore, the efficacies of existing treatments vary, and all have associated safety concerns [8-10]. Systemic glucocorticoids are the primary treatment for complicated IHs, with interferon- $\alpha$ and vincristine used for lesions that do not respond to glucocorticoid therapy $[10,11]$.

In 2008, Leaute-Labreze et al. [12] reported the first case in which propranolol was administered for the treatment of obstructive hypertrophic myocardiopathy in a 1-month-old infant who had undergone treatment with steroids. Thereafter, treating IH with propranolol was deemed effective, with a negligible frequency of side effects. Propranolol is a nonselective betablocker that is used in patients of all ages with hypertension and various cardiac diseases. It is also known to be safe for administration in infants [13].

The proposed mechanisms of the effects of propranolol on $\mathrm{IH}$ include the inhibition of tumor growth and angiogenesis, as well as vasoconstriction [14]. Its known side effects include hypotension, bradycardia, bronchospasm, hypoglycemia, and diarrhea [12]. However, studies of the effects of propranolol on infant growth and development after its prolonged administration are limited, as are studies of the acute side effects mentioned above $[15,16]$. The optimal duration of propranolol treatment in cases of IH has not yet been established; its long-term administration ( $>1$ year) may be required, depending on tumor size and treatment response. Many studies of IH have been published, but few have reported objective measures of treatment response. We thus measured the change of color and size of the lesions to evaluate their response to treatment. We also describe our experience with propranolol dosage and side effects in 23 $\mathrm{IH}$ patients who were treated with propranolol.

\section{METHODS}

Research ethics approval was obtained from the Institutional Review Board at Severance Hospital (Approval No. 4-20171158). Informed consent was obtained from all patients or their representatives for inclusion in this study.

We evaluated 29 infants with newly diagnosed IH who were given propranolol at a dosage of $3 \mathrm{mg} / \mathrm{kg} /$ day, given as two divided doses, between 2015 and 2017, at a tertiary care hospital. Six patients were excluded from the final analysis because of a lack of follow-up visits.

In a retrospective study based on computerized medical records, the charts were reviewed for sex, age, location of the hemangioma, response to treatment as determined by the analysis of photos, age of the patient at the beginning of therapy, duration of therapy, complications, and adverse effects. We used propranolol after an evaluation of the cardiac status of each infant for the treatment of IH in an outpatient clinic.

To assess cardiac status for pediatric cardiac outpatient care, electrocardiography and echocardiography were conducted. Additional imaging studies (ultrasonography or MRI) were conducted if necessary.

The oral administration of propranolol was begun during the next outpatient clinic visit after the evaluation of cardiac status and imaging studies were complete. Patients' caregivers were instructed to cease administering propranolol if severe coughing, dyspnea, vomiting, or diarrhea developed during treatment. Follow-up visits were initially scheduled 2 weeks after the initiation of therapy and then monthly thereafter. Visits were also encouraged in case of any complications. We determined that the duration of treatment would be dependent on the response to treatment.

Patients were evaluated regularly in the outpatient clinic. Patients were photographed prior to the administration of propranolol and at 3, 6, 9, and 12 months after treatment, with a commercial digital camera and a digital camera equipped with the Antera 3D system (Miravex, Dublin, Ireland), which enables analyses of size and color from the chromatic aberration values ( $\mathrm{L}$, $\mathrm{a}$, and $\mathrm{b}$ values). These values express color as three numerical values: $L$ for lightness and $a$ and $b$ for the green-red and blueyellow color components, respectively [17]. The a-value was measured in our study for assessing the redness of the lesions. To assess general aesthetic outcomes and the overall improvement in the appearance of the hemangioma after treatment, we measured and compared the initial and final a-values and the size of the hemangiomas.

We further analyzed changes in color and size using four variables: sex, number of hemangiomas, location of the hemangio- 
ma, and the age of patients at the start of treatment. The starting age was divided into two groups: before and after 6 months after birth. The location of hemangiomas was also divided into two groups: the head and neck group and the non-head and neck group. Furthermore, patients were divided into two groups by the number of hemangiomas: group 1 included patients with only a single hemangioma, whereas group 2 included patients with two or more hemangiomas.

Statistical tests were used to analyze the results. The normality of the distribution of the groups was assessed using the ShapiroWilk test. Statistical analyses were performed using the t-test for independent samples and the Mann-Whitney U-test. A P-value less than 0.05 was considered to indicate statistical significance, and statistical analyses were performed using SPSS 15.0.1 for Windows (SPSS Inc., Chicago, IL, USA).

\section{RESULTS}

In the present study, we included 23 children who were diagnosed as having $\mathrm{IH}$ and who were referred for propranolol treatment. The mean age of the patients at the initiation of treatment was $6.5 \pm 3.6$ months. The average follow-up period was $7.0 \pm$ 3.2 months. There were three male and 20 female patients. Common locations were the head and neck (13 cases, $56.5 \%$ ), trunk (four cases, 17.4\%), extremities (three cases, 13.0\%), and combined locations (three cases, 13.0\%). We divided patients into two groups based on the number of hemangiomas. Group 1 , which included patients with only a single hemangioma, comprised 19 patients, whereas group 2, which included patients with two or more hemangiomas, was composed of four patients (Figs. 1 and 2).

The response to treatment was assessed in terms of color and size using the Antera 3D system (Miravex). At the time of the initial diagnosis, the average measured a-value was 39.7 \pm 7.9 . The average final a-value was $17.0 \pm 14$.5. The difference between the two values was calculated to assess the response of treatment in terms of color fading. The mean change of the avalue was $22.7 \pm 15.0$. Overall, the a-value decreased.

The Shapiro-Wilk test was performed to test the normality of the size-related data. The P-value was less than 0.05 , so we concluded that the data were not normally distributed. The median initial size of the IHs was $115.0 \mathrm{~mm}^{2}$ (range, $4.0-1,517.0 \mathrm{~mm}^{2}$ ) and the median final size was $56.0 \mathrm{~mm}^{2}$ (range, $1.0-891.0$ $\mathrm{mm}^{2}$ ). The percent change in size was calculated as the difference between the initial size and the final size divided by the initial size. The median percent change in size was $32.3 \%$ (range, 1.1\%-92.6\%) (Table 1).

When color fading was analyzed by sex, no significant difference was found in the initial average between male patients $(46.1 \pm 1.1)$ and female patients $(38.8 \pm 8.1)(\mathrm{P}>0.05)$. How-

\section{Fig. 1. Infantile hemangioma on the anterior neck}

Patient 3. (A) At 3 months of age, 1 day prior to treatment with 3 $\mathrm{mg} / \mathrm{kg} /$ day of propranolol. (B) Two months after propranolol treatment. (C) Six months after propranolol treatment. (D) Ten months after propranolol treatment.
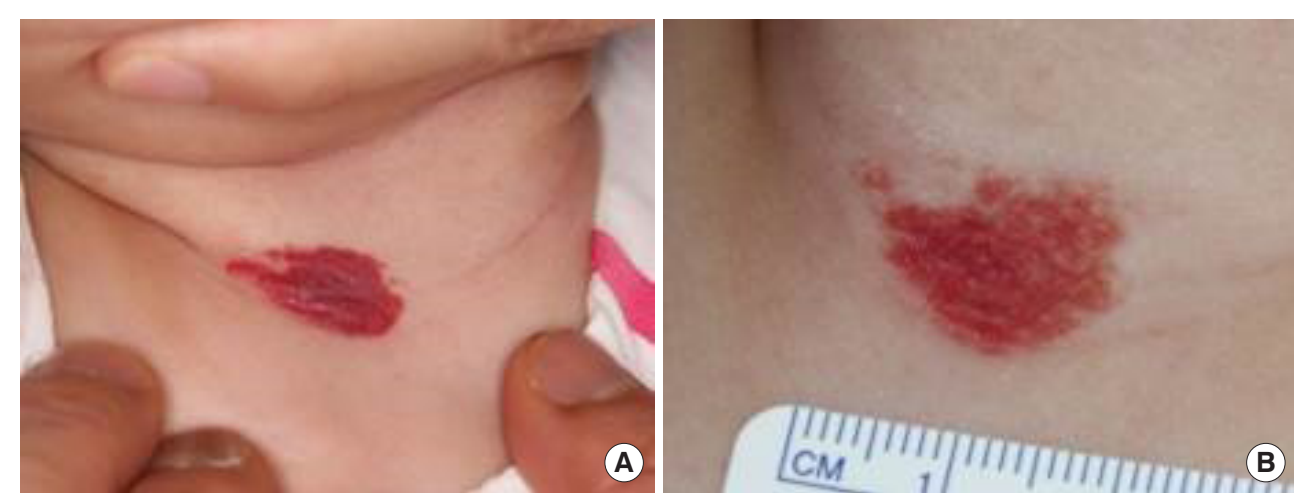

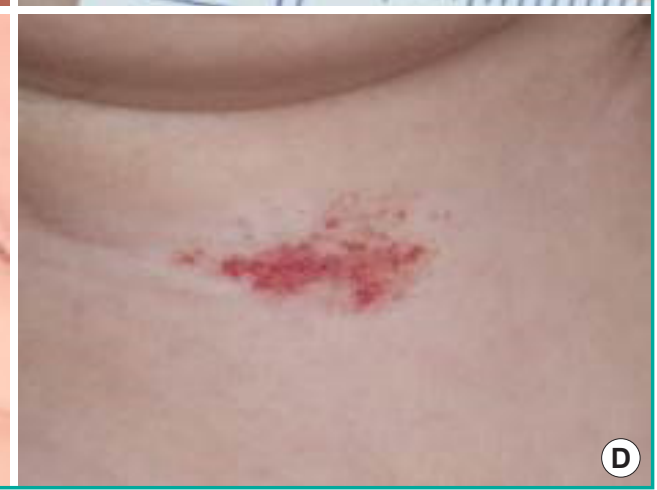




\section{Fig. 2. Infantile hemangioma on the right cheek}

Patient 4. (A) At 3 weeks of age. (B) Three months after the initiation of therapy with $3 \mathrm{mg} / \mathrm{kg} / \mathrm{day}$ of propranolol. (C) Six months of age. (D) Residual hemangioma at 11 months of age.
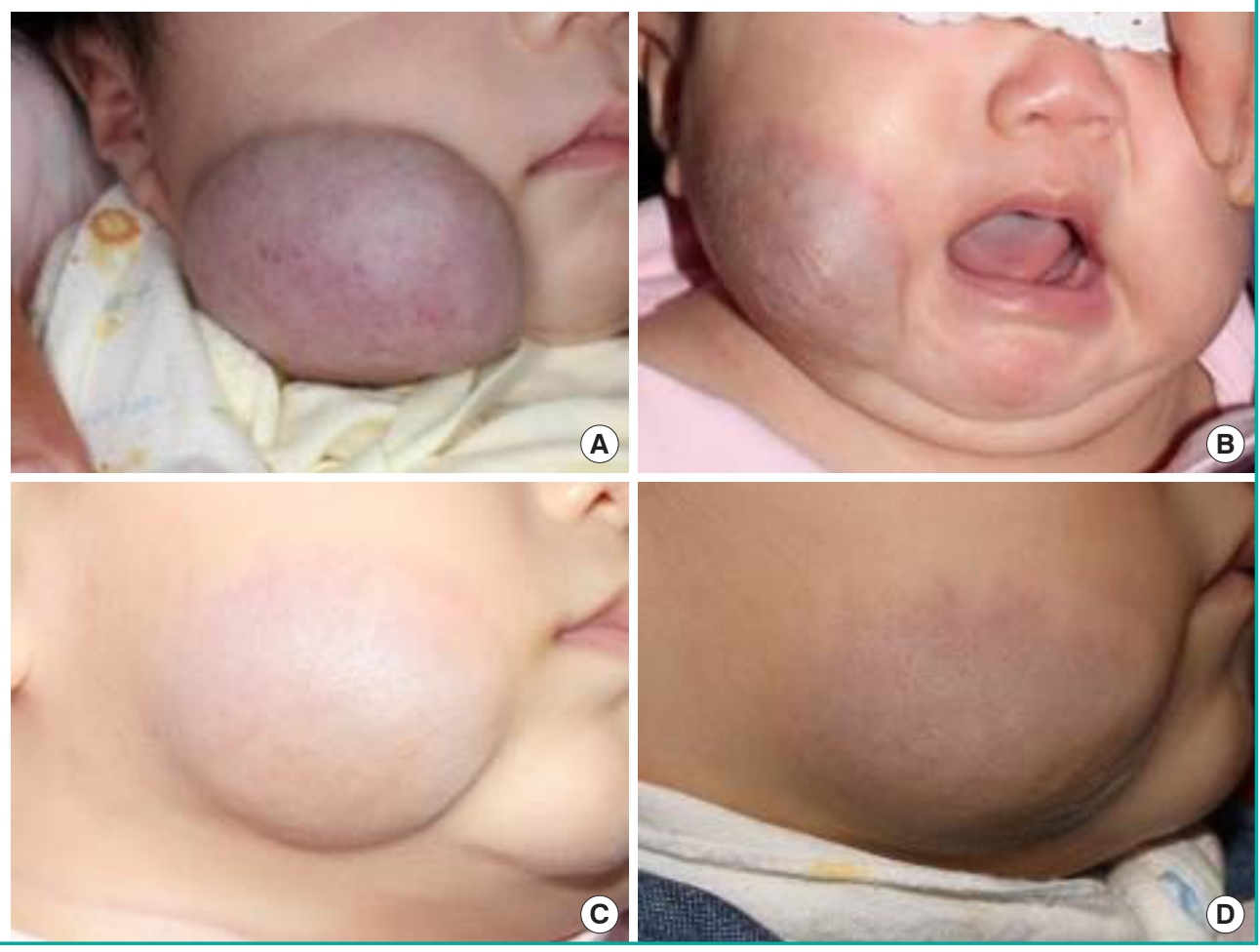

ever, there was a statistically significant difference according to sex in the final a-value, indicating color, and the color change. In male patients, the final a-value was lower $(\mathrm{P}<0.001)$ and the color change was greater than in female patients $(\mathrm{P}<0.001)$. Similar results were found for the number of hemangiomas; group 1 showed a lower final a-value $(\mathrm{P}<0.001)$ and a greater color change than group $2(\mathrm{P}<0.001)$. No other statistically significant differences were found in the analyses according to the location of the hemangioma and the age of patients at the start of treatment (Table 2).

In an analysis of the response to treatment based on size change according to the number of hemangiomas, group 1 showed a significantly greater size change (median, $49.5 \%$; range, $1.1 \%-92.6 \%$ ) than group 2 (median, $12.1 \%$; range, $4.0 \%-16.0 \%$ ) Additionally, the change in size in patients who started treatment before 6 months of age (median, 62.3\%; range $3.0 \%-93.0 \%$ ) was greater than in their counterparts (median, $15.8 \%$; range, $1.0 \%-79.0 \%$; $<0.001$ ). No other statistically significant differences were found in the analyses according to sex and the location of the hemangioma (Table 3).

No severe adverse events were noted in any patient. We observed mild adverse effects (diarrhea or mild fever) that were tolerated without the need to discontinue treatment (Table 4).

\section{DISCUSSION}

Propranolol is widely used for the effective treatment of $\mathrm{IH}$, with a good response rate compared with prednisolone, which is the conventional treatment [18]; in our experience, it also has a good safety profile. Propranolol was the first $\beta$-adrenergic receptor antagonist introduced for the treatment of angina. It is known to reversibly bind $\beta 1$ and $\beta 2$ receptors, reducing heart rate and cardiac output, and causing peripheral vasoconstriction soon after administration [19]. Although the mechanism of the effects of propranolol on IH remain unclear, some studies have reported that vasoconstriction plays a major role in lesion improvement [20], appearing to soften lesions and make them less livid. Moreover, the late effects of propranolol, which are evident several days after administration, include lesion improvement via the inhibition of proangiogenic signals, such as vascular endothelial growth factor and basic fibroblast growth factor, and the stimulation of microvascular endothelial cell apoptosis [21].

The determination of the therapeutic dose of propranolol appears to differ for each hospital. According to Kim et al. [22], treatment with propranolol generally begins at admission, with $0.5 \mathrm{mg} / \mathrm{kg} /$ day administered on the first day of treatment, and 1 $\mathrm{mg} / \mathrm{kg} /$ day given on the second day. If no side effects are observed on the third day, the final treatment dose is increased to 2 


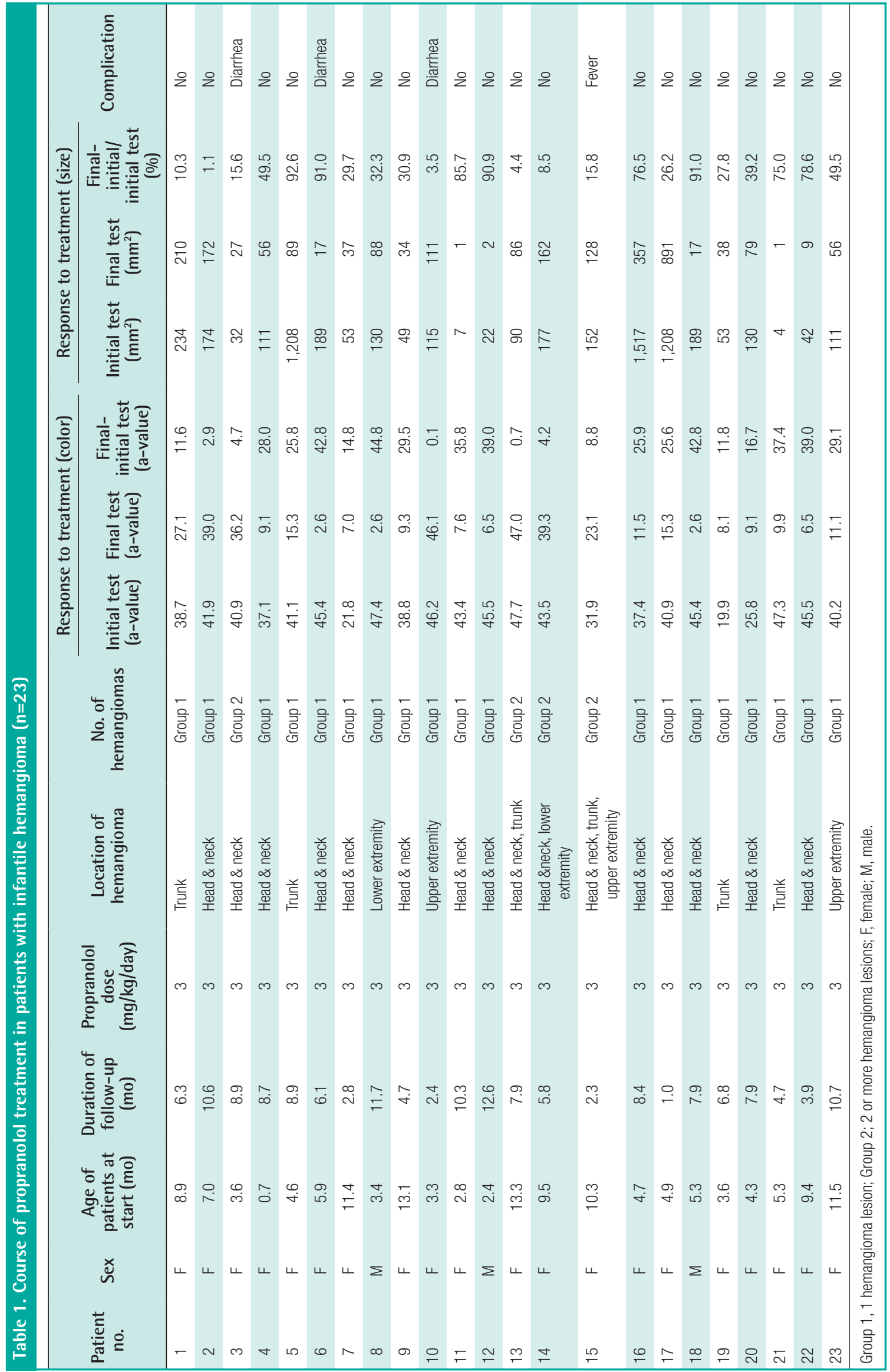




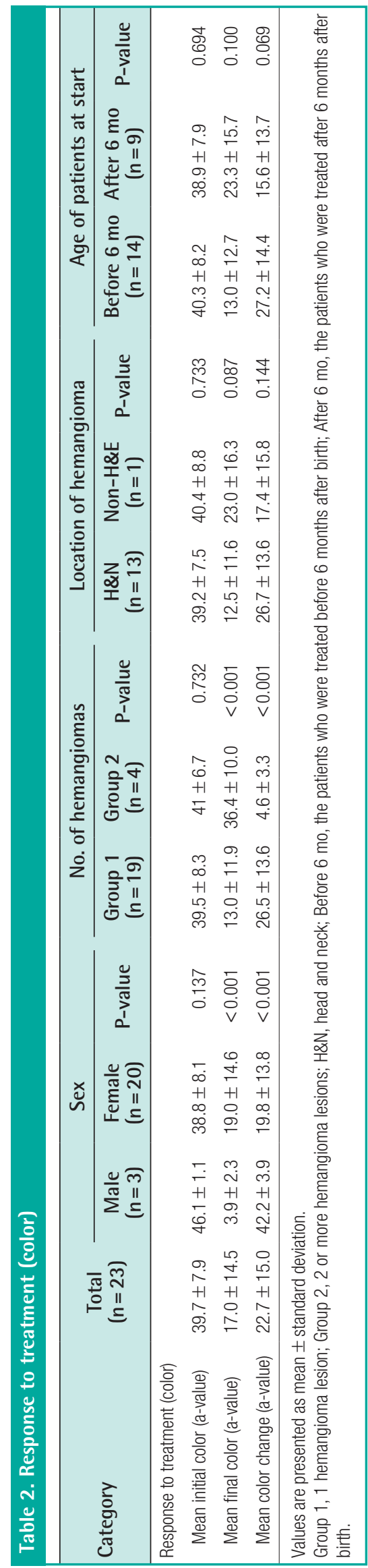
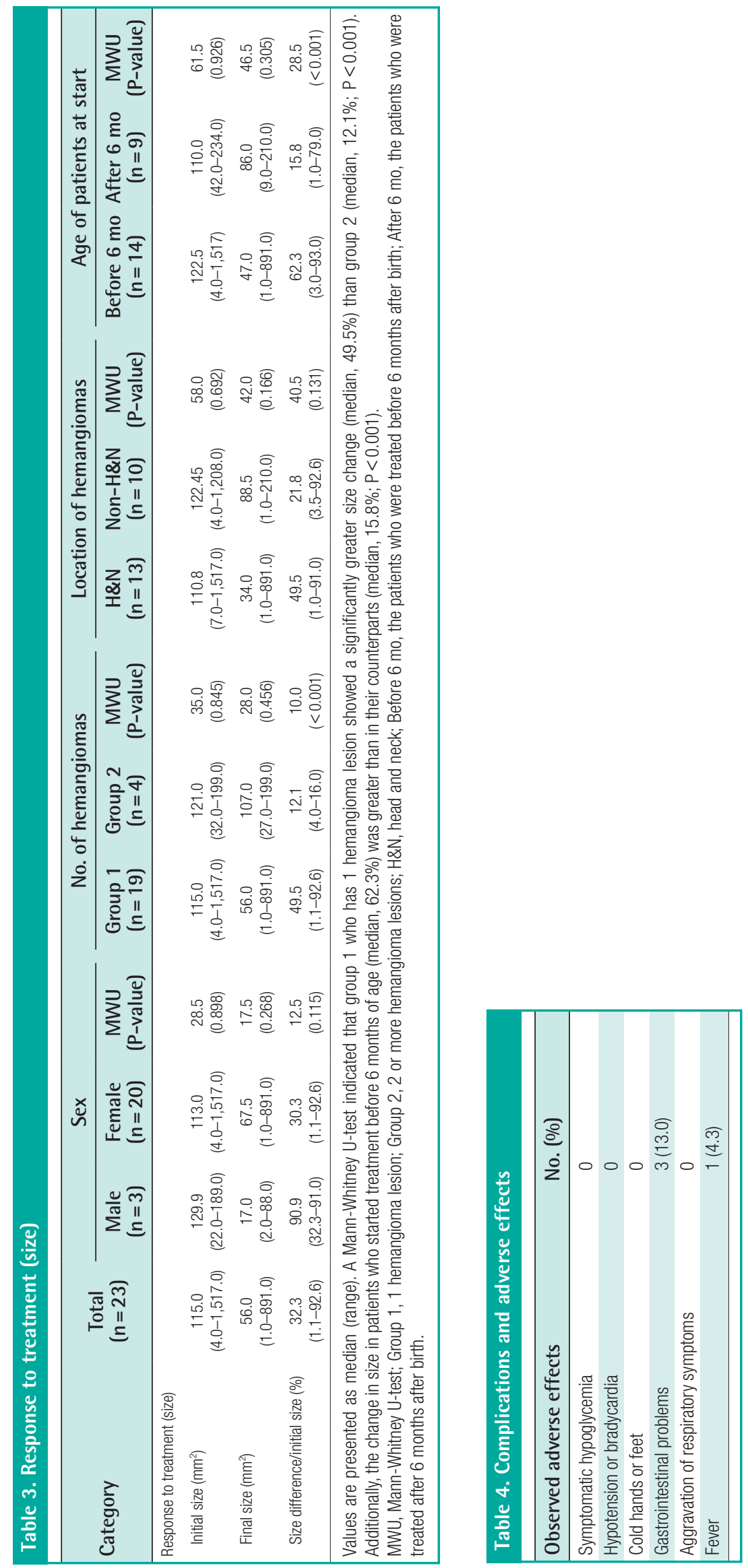
$\mathrm{mg} / \mathrm{kg} /$ day (oral administration 3 times per day every 8 hours), and the patient is discharged. Schupp et al. [1] reported that patients admitted to the hospital were given a dose of $1 \mathrm{mg} / \mathrm{kg} /$ day for 3 days. If no specific adverse events were observed, the dose was increased to $2 \mathrm{mg} / \mathrm{kg} /$ day, and the patient was discharged with this final dose. In other words, it is common to begin with a small dose of the drug initially and to gradually increase the dose over time. Leaute-Labreze et al. [12] reported that their most effective treatment involved a 6-month administration of propranolol at a dose of $3 \mathrm{mg} / \mathrm{kg} /$ day; thus, we prescribed $3 \mathrm{mg} / \mathrm{kg} /$ per day as our initial dose. In our experience, there were no major side effects in the 23 patients, except for 3 cases with minor problems such as diarrhea and fever.

In a large study of 1,264 IHs by Wedgeworth et al. [23], electrocardiography and echocardiography were not necessary for the detection of cardiovascular side effects during treatment with propranolol. Leaute-Labreze et al. [12] reported that adverse events related to propranolol (hypoglycemia, hypotension, bradycardia, and bronchospasm) did not occur frequently, with no significant differences observed between the placebo and treatment groups. However, Holland et al. [16] reported a case of hypoglycemia and recommended the discontinuation of propranolol therapy when accompanied by reduced dietary intake.

Noticeably, previous studies used subjective methods to assess the perceptible aesthetic treatment results. The reliability of subjective assessments is affected by several factors, such as the evaluator's prejudices, variation in lighting conditions, and even the emotional status of the infant. Thus, a subjective analysis can produce only partially reliable clinical implications. In our series, we used the objectively measured parameters of color difference and size, which were calculated with a digital camera and the Antera 3D system.

Interestingly, the response to propranolol was significantly lower in patients with a larger number of lesions than in patients with a smaller number of lesions in terms of color and size change. This finding suggests that propranolol does not effectively inhibit abnormal endothelial cell proliferation and angiogenesis, especially in patients with a large number of hemangiomas.

Male patients showed a greater color change $(\mathrm{P}<0.001)$, whereas there was no sex difference in size changes $(\mathrm{P}<0.001)$. The age at the start of treatment did not show a significant relationship with color change $(\mathrm{P}<0.001)$, but patients who were treated before 6 months after birth showed a significantly greater size change than their counterparts $(\mathrm{P}<0.001)$.

Corticosteroids were initially discovered to induce the accelerated regression of proliferating IHs in the 1960s $[10,24]$ and have been considered the first-line treatment of $\mathrm{IH}$ for over 40 years [25]. However, because of recent clinical successes using the beta-blocker propranolol, the recommended first-line treatment for these lesions is changing.

Izadpanah et al. [18] compared propranolol and corticosteroids in terms of their treatment response rates, demonstrating that the response to propranolol was significantly better $(97.3 \%$ vs. $71.0 \%$, respectively; $\mathrm{P}<0.001$ ), consistent with our study findings that 21 out of 23 patients responded to the treatment (91.3\%). In their meta-analysis of results related to corticosteroid use, the most common side effects reported were altered growth $(6 \%)$ and development of a moon face (5\%). Other reported side effects included osteoporosis, fungal infections, and hypertension. Side effects were encountered in 475 of 2,697 patients (17.6\%). Meanwhile, 96 of 699 patients (13.7\%) experienced side effects after propranolol therapy. The complication rate of steroid therapy was thus higher than that associated with propranolol [18]. The side effects observed at our institution were primarily gastrointestinal problems and fever (17.3\%); although this rate was high, these complications may not have been directly related to propranolol treatment.

This study has limitations in that the number of patients in each group was relatively small; the inclusion of additional cases would improve the significance of our findings. In addition, further large-scale studies are needed to clarify the effects of propranolol on infant growth and development after long-term propranolol treatment. Another limitation is a lack of randomized controlled trials comparing propranolol therapy for $\mathrm{IH}$ with a more conservative treatment. Randomized controlled trials with a set dosage, duration of therapy, and appropriate follow-up are necessary to validate the observed therapeutic benefits of propranolol. The examined factors of the follow-up period, age at the initiation of treatment, treatment duration, and patient weight could influence the response to propranolol therapy. It is thus necessary to control for these factors in future studies.

In conclusion, propranolol is efficacious and has a good safety profile for the treatment of $\mathrm{IH}$. A short duration of treatment ( $<6$ months) at a dose of $3 \mathrm{mg} / \mathrm{kg} /$ day is able to stabilize and induce regression in certain $\mathrm{IH}$ patients. In patients with a single lesion, the response to treatment was better in terms of color fading and size reduction. Furthermore, male patients responded better to propranolol treatment in terms of color fading than female patients, and starting treatment before 6 months after birth was more advantageous for size reduction.

\section{NOTES}

\section{Conflict of interest}

No potential conflict of interest relevant to this article was reported. 


\section{Ethical approval}

The study was approved by the Institutional Review Board of Severance Hospital (IRB No. 4-2017-1158) and performed in accordance with the principles of the Declaration of Helsinki. Written informed consents were obtained.

\section{Patient consent}

The patients provided written informed consent for the publication and the use of their images.

\section{REFERENCES}

1. Schupp CJ, Kleber JB, Gunther P, et al. Propranolol therapy in 55 infants with infantile hemangioma: dosage, duration, adverse effects, and outcome. Pediatr Dermatol 2011;28: 640-4.

2. Haggstrom AN, Drolet BA, Baselga E, et al. Prospective study of infantile hemangiomas: clinical characteristics predicting complications and treatment. Pediatrics 2006;118: 882-7.

3. Ogawa R, Neligan PC. Benign cutaneous and soft-tissue tumors. In: Ogawa R, editor. Benign and malignant nonmelanocytic tumors of the skin and soft tissue. Edinburgh: Elsevier Health Sciences; 2018. p. 707-42.

4. Dubois J, Alison M. Vascular anomalies: what a radiologist needs to know. Pediatr Radiol 2010;40:895-905.

5. Nakayama H. Clinical and histological studies of the classification and the natural course of the strawberry mark. J Dermatol 1981;8:277-91.

6. North PE, Waner M, Mizeracki A, et al. A unique microvascular phenotype shared by juvenile hemangiomas and human placenta. Arch Dermatol 2001;137:559-70.

7. Sirachainan N, Lekanan W, Thammagasorn Y, et al. Response to propranolol in infantile hemangioma. Pediatr Int 2016;58:662-5.

8. Barrio VR, Drolet BA. Treatment of hemangiomas of infancy. Dermatol Ther 2005;18:151-9.

9. Enjolras O, Breviere GM, Roger G, et al. Vincristine treatment for function- and life-threatening infantile hemangioma. Arch Pediatr 2004;11:99-107.

10. Zarem HA, Edgerton MT. Induced resolution of cavernous hemangiomas following prednisolone therapy. Plast Reconstr Surg 1967;39:76-83.

11. Chiller KG, Passaro D, Frieden IJ. Hemangiomas of infancy: clinical characteristics, morphologic subtypes, and their relationship to race, ethnicity, and sex. Arch Dermatol 2002; 138:1567-76.

12. Leaute-Labreze C, Hoeger P, Mazereeuw-Hautier J, et al. A randomized, controlled trial of oral propranolol in infantile hemangioma. N Engl J Med 2015;372:735-46.

13. Leaute-Labreze C, Boccara O, Degrugillier-Chopinet $C$, et al. Safety of oral propranolol for the treatment of infantile hemangioma: a systematic review. Pediatrics 2016;1384: e20160353.

14. Ji Y, Chen S, Xu C, et al. The use of propranolol in the treatment of infantile haemangiomas: an update on potential mechanisms of action. Br J Dermatol 2015;172:24-32.

15. Aletaha M, Salour H, Bagheri A, et al. Oral propranolol for treatment of pediatric capillary hemangiomas. J Ophthalmic Vis Res 2012;7:130-3.

16. Holland KE, Frieden IJ, Frommelt PC, et al. Hypoglycemia in children taking propranolol for the treatment of infantile hemangioma. Arch Dermatol 2010;146:775-8.

17. International Color Consortium. Image technology colour management: architecture, profile format, and data structure. dSpecification ICC. 1: 2004-10 (Profile version 4.2. 0.0) [Internet]. Reston, VA: ICC; c2004 [cited 2018 Nov 7]. Available from http://www.color.org/icc1v42_2006-05.pdf.

18. Izadpanah A, Izadpanah A, Kanevsky J, et al. Propranolol versus corticosteroids in the treatment of infantile hemangioma: a systematic review and meta-analysis. Plast Reconstr Surg 2013;131:601-13.

19. Helfand M, Peterson K. Drug class review on beta adrenergic blockers. Portland: Oregon Health \& Science University; 2005.

20. Nguyen J, Fay A. Pharmacologic therapy for periocular infantile hemangiomas: a review of the literature. Semin Ophthalmol 2009;24:178-84.

21. Denoyelle F, Leboulanger N, Enjolras O, et al. Role of propranolol in the therapeutic strategy of infantile laryngotracheal hemangioma. Int J Pediatr Otorhinolaryngol 2009;73: 1168-72.

22. Kim KM, Min, DH, Jung, HL, et al. Propranolol as a firstline treatment for pediatric hemangioma: outcome of a single institution over one year. Clinical Pediatric HematologyOncology 2016;23:97-104.

23. Wedgeworth $\mathrm{E}$, Glover $\mathrm{M}$, Irvine $\mathrm{AD}$, et al. Propranolol in the treatment of infantile haemangiomas: lessons from the European Propranolol in the Treatment of Complicated Haemangiomas (PITCH) Taskforce survey. Br J Dermatol 2016;174:594-601.

24. Fost NC, Esterly NB. Successful treatment of juvenile hemangiomas with prednisone.J Pediatr 1968;72:351-7.

25. Boon LM, MacDonald DM, Mulliken JB. Complications of systemic corticosteroid therapy for problematic hemangioma. Plast Reconstr Surg 1999;104:1616-23. 